\title{
Vibração e ruído emitidos por dois tratores agrícolas
}

\author{
Vibration and noise levels emitted by two tractors \\ João Paulo Arantes Rodrigues da Cunha ${ }^{1 *}$, Marcus Antonio Viana Duarte ${ }^{2}$, \\ Cristiano Márcio Alves de Souza ${ }^{3}$
}

\section{RESUMO}

A operação de tratores agrícolas impõe grande estresse físico e mental aos tratoristas. Este trabalho teve como objetivo avaliar os níveis de vibração e ruído emitidos por dois tratores agrícolas, defasados temporalmente, em diferentes rotações do motor (1700, 1850 e $2000 \mathrm{rpm}$ ). Nas avaliações foram utilizados um trator de $60,35 \mathrm{~kW}$, fabricado em 1988, e um trator de $89,06 \mathrm{~kW}$, fabricado em 2009, ambos não-cabinados e tracionando um arado de discos. As avaliações de ruído foram feitas com um medidor de pressão sonora (decibelímetro), no posto de operação da máquina. As avaliações de vibração foram feitas no assento do trator, utilizando um sistema de aquisição automática de dados com três acelerômetros, nos sentidos vertical e horizontal. Os resultados permitiram as seguintes conclusões: a operação de aração com os dois tratores apresentaram níveis de ruído acima do limite de 85 dB (A) para 8 horas de exposição diária, estabelecido pela NR-15, fazendo-se necessário o uso de dispositivos de proteção auricular durante sua execução. O trator mais antigo apresentou níveis de vibração que podem comprometer a saúde, segurança, conforto e eficiência do tratorista. O mesmo não ocorreu para o trator mais novo.

Palavras-chave: máquinas agrícolas, ergonomia, saúde ocupacional.

\begin{abstract}
Tractor driving imposes a lot of physical and mental stress upon the operator. The aim of this work was to evaluate the vibration and noise levels emitted by tractors with different ages, under different motor rotations (1700, 1850 and $2000 \mathrm{rpm})$. In the evaluations, two agricultural tractors (a 1988 model year with $60.35 \mathrm{~kW}$ of power and a 2009 model year with $89.06 \mathrm{~kW}$ ), without cabin were used, working with a plough. The noise evaluations were made with a sound level meter, in the driver's position. The vibration evaluations were made on the driving seat using an automatic acquisition data system with three accelerometers, in the vertical and horizontal planes. The results showed that the service with the tractors presented noise levels higher than the $85 \mathrm{~dB}(\mathrm{~A})$ limit for 8 hours of daily exposure, established by the Brazilian Legislation on insalubrity. The need of hearing protection device use by the machine operators was observed. The older tractor presented vibration levels that could compromise health, safety, comfort and efficiency of the driver. This not occurs for the newer tractor.
\end{abstract}

Key words: agricultural machines, ergonomics, occupational healthy.

\section{Introdução}

O problema da transmissão da vibração dos tratores agrícolas aos seus operadores vem sendo pesquisado há anos. Contudo, ainda existe uma clara necessidade de desenvolver sistemas para sua prevenção (Tiemessen et al., 2007). Devido à inexistência de amortecedores e molas, como em veículos urbanos, o assento do tratorista tem importante papel em minimizar essa vibração, sendo, portanto, importante componente do trator (Jain et al., 2008). De acordo com Tewari e Dewangan (2009), a redução da vibração, além de diminuir o estresse do operador, permite melhor qualidade de vida e aumento da jornada de trabalho sem causar excesso de fadiga. Bluthner et al. (2006) afirmam que a vibração pode levar a sérios danos a espinha dorsal dos operadores.

Nas décadas passadas pouca atenção foi dada a esse problema, contudo, com a introdução da restrição a exposição do trabalhador a níveis excessivos de vibração na legislação da Comunidade

1 Universidade Federal de Uberlândia, Campus Umuarama, Instituto de Ciências Agrárias, Uberlândia, MG, Brasil.

E-mail: jpcunha@iciag.ufu.br.* Autor para correspondência.

2 Universidade Federal de Uberlândia, Campus Santa Mônica, Faculdade de Engenharia Mecânica, Uberlândia, MG, Brasil.

3 Universidade Federal da Grande Dourados, Faculdade de Ciências Agrárias, Dourados, MS, Brasil.

Fecha de Recepción: 15 Diciembre, 2009.

Fecha de Aceptación: 2 Febrero, 2012. 
Européia, no ano de 2002, a situação alterou-se. Atualmente, a maioria das empresas fabricantes de tratores agrícolas incorpora algum sistema para reduzir a vibração (Scarlett et al., 2007). Marsili et al. (2002) mostraram que a redução da vibração em tratores por meio de sistemas de suspensão pode permitir um incremento superior a $50 \%$ no tempo de exposição do operador ao trabalho.

A operação de tratores impõe grande estresse físico e mental. Se o posto de trabalho do tratorista não for confortável, seu desempenho e segurança poderão ser comprometidos (Debiasi et al., 2004 e Mehta et al., 2008). Além da vibração, o ruído também pode comprometer a saúde do trabalhador. Segundo Dewangan et al. (2005), a exposição durante 40 horas por semana a níveis de ruído de $85 \mathrm{~dB}(\mathrm{~A})$ é considerada segura, mas níveis acima desse limite podem causar hipoacusia. Ainda segundo esses autores, apesar do ruído já estar sendo estudado há vários anos nos tratores agrícolas, o problema ainda persiste em alguns equipamentos fabricados na atualidade. Souza et al. (2004) e Depczynski et al. (2005) também mostram que o ruído ainda é um problema a ser resolvido no meio rural.

A mitigação do problema da vibração e do ruído está ligada à redução da intensidade dos mesmos na fonte, à diminuição do tempo de exposição ou ao uso de equipamentos de proteção individual, no caso do ruído. Dessa forma, é importante conhecer a realidade das máquinas atuais em termo de vibração e ruído para planejar a melhor estratégia de combate ao problema.

Este trabalho teve como objetivo avaliar os níveis de vibração e ruído emitidos por dois tratores agrícolas, defasados temporalmente, em diferentes rotações do motor, comparando com as normas vigentes.

\section{Material e Métodos}

Os ensaios de avaliação dos níveis de vibração e ruído emitidos por dois conjuntos trator-implemento foram realizados em área experimental da Fazenda Capim Branco, pertencente a Universidade Federal de Uberlândia, localizada no município de Uberlândia, MG, Brasil. O solo do local é classificado como Latossolo Vermelho Distroférrico (Embrapa, 1999), com textura superficial muito argilosa e topografia plana. No momento do ensaio, o solo apresentava teor de água de $19,6 \%$ e vegetação espontânea, constituída principalmente por Brachiaria decumbens.
As avaliações foram feitas em dois tratores distintos, trabalhando a três rotações do motor $(1700,1850$ e $2000 \mathrm{rpm})$ e tracionando um arado fixo de três discos lisos (26"), com 0,9 m de largura de corte. A aração foi feita a $20 \mathrm{~cm}$ de profundidade e com $5 \mathrm{~km} \mathrm{~h}^{-1}$ de velocidade de deslocamento, com intuito de simular a mesma carga nos dois conjuntos, independente da potência dos motores. Empregou-se um trator agrícola (A) fabricado em 1988 , com motor do ciclo Diesel de quatro cilindros com potência de $60,35 \mathrm{~kW}(82 \mathrm{cv}$ ) e um trator agrícola (B) com tração dianteira auxiliar, fabricado em 2009 (100 horas de uso), com motor do ciclo Diesel de seis cilindros com potência de $89,06 \mathrm{~kW}$ $(121 \mathrm{cv})$. Ambos os tratores apresentavam assentos originais de fábrica.

O trator A apresentava pneus traseiros Pirelli TM 95-18,4-34, juntamente com dois lastros de 50 $\mathrm{kg} \mathrm{e} 3 / 4$ de água em cada pneu, à pressão de $97 \mathrm{kPa}$ (14 psi), e pneus dianteiros Pirelli TD 500-7,50-18, à pressão de $276 \mathrm{kPa}$ (40 psi), com 6 lastros metálicos frontais de $15 \mathrm{~kg}$. O trator B apresentava pneus traseiros Pirelli TM 98-23,1-30, com $80 \mathrm{~kg}$ de lastro e 3/4 de água em cada pneu, à pressão de $97 \mathrm{kPa}(14$ psi), e pneus dianteiros Goodyear Dynatorque II14,9-26, também com $80 \mathrm{~kg}$ de lastro e 3/4 de água em cada pneu, à pressão de $97 \mathrm{kPa}$ (14 psi),

Antes do início de cada ensaio, foi medido o ruído de fundo, a velocidade do vento, a umidade relativa e a temperatura do ar. Foi utilizado um protetor de ventos, junto ao microfone do medidor de pressão sonora, com o intuito de uniformizar as condições de leitura e evitar a influência de possíveis rajadas de vento.

Foi utilizado um medidor de pressão sonora (Decibelímetro 2236, Bruel \& Kjaer) calibrado anteriormente (Calibrador de microfones CAL200, Larson Davis), no circuito de resposta lenta (slow) e curva de ponderação "A". As leituras foram realizadas próximas ao ouvido do operador, no lado correspondente ao escapamento de saída dos gases do motor. O tempo de aquisição para integração dos dados foi de 10 minutos, em situação semelhante de trabalho, para cada condição.

Foram determinados: L10, L50, L90, Leq e nível máximo de ruído. Os níveis denominados L10, L50 e L90 representam os valores acima dos quais os demais níveis permanecem $10 \%, 50 \%$ e $90 \%$ do tempo total, respectivamente. O nível sonoro médio integrado durante os dez minutos avaliados é denominado nível sonoro equivalente (Leq). Este 
último foi submetido à análise de regressão em função da rotação do motor. A partir dos valores de Leq, foram analisados os tempos de exposição máxima que o operador poderia ficar exposto, sem o uso de protetores auriculares, de acordo com a NR-15 (Brasil, 2009).

Com relação à avaliação de vibração, a grandeza primária utilizada foi a aceleração, expressa em m s${ }^{-2}$, baseada na norma ISO 2631 (ISO, 1978). Foram utilizados três acelerômetros (PCB, sendo dois 352C67 e um 352C22), um condicionador de sinal e um sistema de aquisição automática de dados, composto por um conversor A/D (National Instruments, tipo USB 9233) e um microcomputador. Os três acelerômetros foram previamente calibrados utilizando um calibrador de acelerômetros (Tipo 4294, Bruel \& Kjaer) Após a aquisição, os dados foram processados utilizando o software AQUIS IV-CVRI, e os resultados da raiz média quadrática (RMS) das acelerações das vibrações determinados em função da frequiência em bandas de $1 / 3$ de oitava. $\mathrm{O}$ valor RMS ou valor eficaz, que é a raiz quadrada dos valores quadrados médios das acelerações, é a mais importante medida da amplitude porque mostra a média da energia contida no movimento vibratório.

Os acelerômetros foram posicionados junto a uma placa metálica colocada entre o assento original do trator e o tratorista. Dois acelerômetros foram colocados na horizontal defasados de $90^{\circ}$ (sentido do deslocamento do trator - ay e transversal ao deslocamento - ax) e um na vertical (sentido az). O tempo total de aquisição foi de 10 minutos, com o trator operando em condições semelhantes, sendo que os sinais foram adquiridos com uma freqüência de aquisição de $4096 \mathrm{~Hz}$. As acelerações em bandas de $1 / 3$ de oitava, centradas entre 1 e $100 \mathrm{~Hz}$, foram estimadas via análise espectral utilizando 8192 pontos com uma sobreposição de $50 \%$. Os resultados obtidos foram comparados com os limites de exposição estabelecidos pela norma ISO 2631 (ISO, 1978).

\section{Resultados e Discussão}

Os resultados dos níveis de ruído emitidos pelos tratores estão expostos na Figura 1. Em todos os resultados analisados, não foi necessário fazer correção do ruído de fundo, uma vez que o mesmo ficou em torno de $30 \mathrm{~dB}(\mathrm{~A})$ abaixo dos níveis de ruído gerados pelo trator e implemento.

De maneira geral, os valores dos níveis sonoros distribuídos no tempo (L10, L50 e L90) estiveram próximos, sendo o nível sonoro equivalente, então, uma boa medida para estudo do potencial de lesão auditiva. Em todas as condições estudadas, independente do trator, o Leq foi superior a $85 \mathrm{~dB}(\mathrm{~A})$, que é a exposição máxima permitida para uma jornada de trabalho de oito horas, o que demonstra que o avanço tecnológico não foi suficiente para reduzir o problema do ruído nos tratores não-cabinados. Destaca-se que Franklin et al. (2006) mostraram que as cabines nos tratores podem reduzir o nível de ruído em $16 \mathrm{~dB}$ (A). Ainda de acordo com estudo realizado por esses autores, tratores velhos produziram $6 \mathrm{~dB}(\mathrm{~A})$ a mais que tratores novos.

Vale ressaltar também que os valores máximos encontrados para o trator A, em algumas condições, estiveram acima de $115 \mathrm{~dB}$ (A), valor limite para trabalho sem protetor auricular (Brasil, 2009).
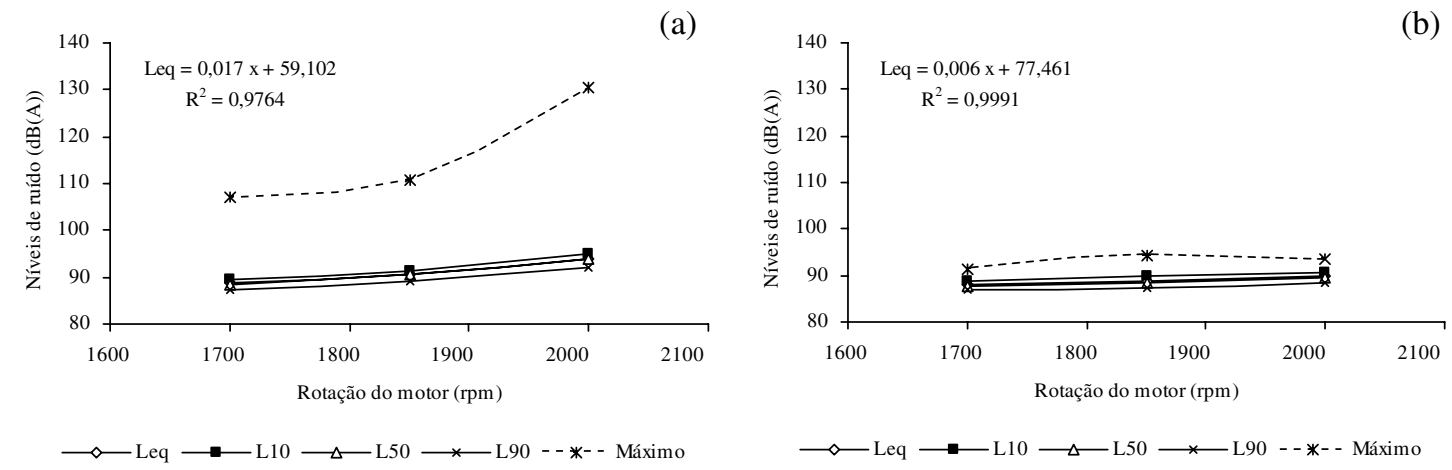

Figura 1. Níveis de ruído (dB(A)) emitido pelo trator A de 1988 (a) e B de 2009 (b), durante a operação com arado de discos, trabalhando em diferentes rotações do motor. Noise levels (dB(A)) emitted by the tractor A (1988) (a) and tractor B (2009) (b), working with a plough, under different motor rotations. 
Trabalhando com rotação do motor de $2000 \mathrm{rpm}$, o conjunto apresentou pico de pressão sonora de 130,4 dB (A). Com o trator B, o pico de ruído foi de 94 dB (A), demonstrando uma redução de valores com a evolução tecnológica dos tratores. $\mathrm{O}$ aumento da rotação de trabalho do trator levou ao incremento dos níveis de ruído, concordando com os dados apresentados por Dewangan et al. (2005).

Na Tabela 1, é apresentado o tempo laborativo máximo diário permitido para um trabalhador, considerando-se o nível sonoro equivalente, de acordo com cada situação de trabalho avaliada, e a falta de protetor auricular, levando-se em conta a norma NR-15 (Brasil, 2009). Analisando-se os valores encontrados, percebe-se a necessidade de conscientização dos operadores quanto à importância da utilização dos equipamentos de proteção individual, e também daqueles que trabalham próximos às máquinas.

Tabela 1. Tempos de exposição diária máxima permissível, de acordo com os níveis de ruído emitidos pelos tratores durante a operação com arado de discos, operando em diferentes rotações do motor. Daily exposition time according to noise levels emitted by the tractors, working with a plough, under different motor rotations.

\begin{tabular}{ccl}
\hline Trator & $\begin{array}{c}\text { Rotação do motor } \\
\text { (rpm) }\end{array}$ & $\begin{array}{l}\text { Tempo de } \\
\text { exposição }\end{array}$ \\
\hline & 1700 & 4 h e 30 min \\
A & 1850 & 3 h e 30 min \\
(Ano 1988) & 2000 & $2 \mathrm{~h} \mathrm{e} 15 \mathrm{~min}$ \\
\hline & 1700 & $5 \mathrm{~h}$ \\
B & 1850 & $4 \mathrm{~h} \mathrm{e} \mathrm{30} \mathrm{min}$ \\
& 2000 & $4 \mathrm{~h}$ \\
\hline
\end{tabular}

Apesar de alguns modelos de protetor auricular permitirem redução de ruído superior a $20 \mathrm{~dB}(\mathrm{~A})$, Pessina e Guerretti (2000), avaliando a eficiência de vários dispositivos para a redução de ruído no ouvido de operadores de tratores agrícolas, concluíram que, em média, os dispositivos de proteção auricular permitem uma atenuação do nível de ruído na ordem de $10 \mathrm{~dB}(\mathrm{~A})$. Considerando esse valor, é possível perceber que, de fato, os níveis de ruído observados em algumas condições encontram-se muito elevados. Dependendo da qualidade do protetor auricular e da forma e tempo de utilização, mesmo com o emprego desse dispositivo, poderá haver desconforto e dano à saúde do trabalhador. Outra constatação importante refere-se a importância da cabine para o ganho de qualidade no ambiente laboral, já que mesmo em um trator novo o nível de ruído foi alto.

Os valores de vibração (bandas de 1/3 de oitava) medidos para as três direções estão mostrados nas Tabelas 2 e 3. Para fins de comparação, nas Figuras 2, $3,4,5,6$ e 7 estão mostrados os valores de aceleração medidos e os valores recomendados pela norma ISO 2631 (ISO, 1978) para limite de exposição (LE), nível de conforto reduzido (NCR) e nível de eficiência reduzida (NER), para oito horas de exposição.

Da análise dos dados do trator A e utilizando os critérios do nível reduzido de eficiência, verificase que os níveis de vibração foram inferiores aos limites estabelecidos para oito horas de trabalho, nos três sentidos avaliados, para o trator operando a $1700 \mathrm{rpm}$. No entanto, a $1850 \mathrm{rpm}$ e $2000 \mathrm{rpm}$, no sentido do deslocamento do trator (ay), os níveis de vibração excedem os limites de exposição diária de 8 horas, chegando em algumas frequiências a valores não aceitáveis para o trabalho. Provavelmente, a somatória da vibração do trator às oscilações do terreno deve ter contribuído para os resultados apresentados. Considerando os critérios do limite de exposição (saúde e segurança), nota-se que o limite de vibração para oito horas diárias foi ultrapassado a 1850 e 2000 rpm, no sentido ay. Levando-se em conta o nível de conforto reduzido, nota-se que nas três rotações analisadas há situações em que a vibração excede o limite para oito horas diárias de trabalho.

Com relação ao trator $\mathrm{B}$, em todos os sentidos (az, ax e ay) e rotações do motor, os níveis de vibração foram inferiores ao permitido pela norma ISO 2631, considerando oito horas de trabalho, para limite de exposição (LE), nível de conforto reduzido (NCR) e nível de eficiência reduzida (NER). O valor máximo detectado de aceleração foi $0,00404 \mathrm{~m} \mathrm{~s}^{-2}$, empregando $2000 \mathrm{rpm}$ no motor, para aceleração vertical. Isso demonstra que o assento, bem como a estrutura do trator, foram capazes de absorver bem os impactos, criando uma boa condição de trabalho para o operador da máquina.

Bouazara et al. (2006) também encontraram redução da exposição a vibração com o uso de assentos mais modernos dotados de suspensão. Contudo, Paddan e Griffin (2002) afirmam que o assento de um veículo pode reduzir como também aumentar a 
Tabela 2. Valores RMS para as acelerações $\left(\mathrm{m} \mathrm{s}^{-2}\right)$ obtidas durante a operação do conjunto trator A (Ano 1988) e arado de discos, nas respectivas rotações do motor. RMS acceleration values $\left(\mathrm{m} \mathrm{s}^{-2}\right)$ during tractor A (1988) operation, under different motor rotations.

\begin{tabular}{|c|c|c|c|c|c|c|c|c|c|}
\hline \multirow[b]{2}{*}{$\begin{array}{l}\text { Freqüência } \\
(\mathrm{Hz})\end{array}$} & \multicolumn{3}{|c|}{$1700 \mathrm{rpm}$} & \multicolumn{3}{|c|}{$1850 \mathrm{rpm}$} & \multicolumn{3}{|c|}{$2000 \mathrm{rpm}$} \\
\hline & $\begin{array}{l}\text { Assento } \\
\text { Vertical } \\
\text { (az) }\end{array}$ & $\begin{array}{c}\text { Assento } \\
\text { Horizontal } \\
\text { (ax) }\end{array}$ & $\begin{array}{c}\text { Assento } \\
\text { Horizontal } \\
\text { (ay) }\end{array}$ & $\begin{array}{c}\text { Assento } \\
\text { Vertical } \\
\text { (az) }\end{array}$ & $\begin{array}{c}\text { Assento } \\
\text { Horizontal } \\
\text { (ax) }\end{array}$ & $\begin{array}{c}\text { Assento } \\
\text { Horizontal } \\
\text { (ay) }\end{array}$ & $\begin{array}{c}\text { Assento } \\
\text { Vertical } \\
\text { (az) }\end{array}$ & $\begin{array}{c}\text { Assento } \\
\text { Horizontal } \\
\text { (ax) }\end{array}$ & $\begin{array}{c}\text { Assento } \\
\text { Horizontal } \\
\text { (ay) }\end{array}$ \\
\hline 1,00 & 0,27610 & 0,0642 & 0,06600 & 0,11310 & 0,09410 & 1,39400 & 0,19500 & 0,12840 & 32,76270 \\
\hline 1,25 & 0,24490 & 0,05930 & 0,06940 & 0,11570 & 0,08800 & 1,00150 & 0,20060 & 0,11390 & 37,67470 \\
\hline 1,60 & 0,16900 & 0,07100 & 0,07480 & 0,13740 & 0,08910 & 0,74620 & 0,21880 & 0,09990 & 38,72280 \\
\hline 2,00 & 0,12250 & 0,04600 & 0,05180 & 0,10370 & 0,05740 & 0,76000 & 0,16300 & 0,06680 & 30,97220 \\
\hline 2,50 & 0,13730 & 0,04160 & 0,04090 & 0,08130 & 0,05320 & 0,61690 & 0,14500 & 0,06050 & 32,56370 \\
\hline 3,16 & 0,14440 & 0,03910 & 0,03460 & 0,06440 & 0,05130 & 0,76110 & 0,13120 & 0,06200 & 24,50160 \\
\hline 4,00 & 0,10260 & 0,03540 & 0,03260 & 0,06140 & 0,04560 & 0,53360 & 0,09770 & 0,04660 & 21,40690 \\
\hline 5,00 & 0,10150 & 0,04510 & 0,04170 & 0,06860 & 0,06020 & 0,56450 & 0,08690 & 0,04640 & 18,63200 \\
\hline 6,00 & 0,05380 & 0,02890 & 0,03420 & 0,05020 & 0,03650 & 0,65990 & 0,08860 & 0,04840 & 18,65460 \\
\hline 8,00 & 0,04410 & 0,02360 & 0,03150 & 0,03530 & 0,02840 & 0,54180 & 0,04650 & 0,03140 & 18,47110 \\
\hline 10,00 & 0,02680 & 0,01920 & 0,02620 & 0,02310 & 0,02530 & 0,50340 & 0,02960 & 0,03640 & 19,95660 \\
\hline 12,50 & 0,02070 & 0,01920 & 0,02240 & 0,01760 & 0,02200 & 0,60770 & 0,02180 & 0,03530 & 19,94880 \\
\hline 16,30 & 0,02520 & 0,01840 & 0,04940 & 0,01830 & 0,01480 & 0,98940 & 0,01980 & 0,02220 & 22,25320 \\
\hline 20,00 & 0,02410 & 0,02060 & 0,03810 & 0,02060 & 0,02130 & 0,97210 & 0,02420 & 0,02700 & 21,12420 \\
\hline 25,00 & 0,09980 & 0,19900 & 0,04950 & 0,10560 & 0,16890 & 0,72920 & 0,02070 & 0,04430 & 19,93660 \\
\hline 31,60 & 0,04260 & 0,05370 & 0,02690 & 0,09400 & 0,14750 & 0,88340 & 0,09760 & 0,32440 & 24,30330 \\
\hline 40,00 & 0,11560 & 0,03370 & 0,07170 & 0,06430 & 0,03570 & 0,89780 & 0,03470 & 0,07060 & 22,37670 \\
\hline 50,00 & 1,58710 & 0,30000 & 0,86400 & 1,02410 & 0,38680 & 1,51140 & 0,10220 & 0,12490 & 27,65320 \\
\hline 63,00 & 0,40030 & 0,10030 & 0,26740 & 0,88330 & 0,37190 & 2,74240 & 0,37730 & 1,32080 & 115,62300 \\
\hline 80,00 & 0,06070 & 0,16860 & 0,40960 & 0,05870 & 0,11610 & 1,46430 & 0,07160 & 0,25630 & 36,29990 \\
\hline 100,00 & 0,11880 & 0,28250 & 0,69260 & 0,11520 & 0,18770 & 1,54480 & 0,05370 & 0,18700 & 30,53920 \\
\hline
\end{tabular}

Tabela 3.Valores RMS para as acelerações $\left(\mathrm{m} \mathrm{s}^{-2}\right.$ ) obtidas durante a operação do conjunto trator B (Ano 2009) e arado de discos, nas respectivas rotações do motor. RMS acceleration values $\left(\mathrm{m} \mathrm{s}^{-2}\right)$ during tractor B (2009) operation, under different motor rotations.

\begin{tabular}{|c|c|c|c|c|c|c|c|c|c|}
\hline \multirow[b]{2}{*}{$\begin{array}{l}\text { Freqüuência } \\
(\mathrm{Hz})\end{array}$} & \multicolumn{3}{|c|}{$1700 \mathrm{rpm}$} & \multicolumn{3}{|c|}{$1850 \mathrm{rpm}$} & \multicolumn{3}{|c|}{$2000 \mathrm{rpm}$} \\
\hline & $\begin{array}{c}\text { Assento } \\
\text { Vertical } \\
\text { (az) }\end{array}$ & $\begin{array}{l}\text { Assento } \\
\text { Horizontal } \\
\quad(a x)\end{array}$ & $\begin{array}{l}\text { Assento } \\
\text { Horizontal } \\
\text { (ay) }\end{array}$ & $\begin{array}{c}\text { Assento } \\
\text { Vertical } \\
\text { (az) }\end{array}$ & $\begin{array}{l}\text { Assento } \\
\text { Horizontal } \\
\quad(a x)\end{array}$ & $\begin{array}{l}\text { Assento } \\
\text { Horizontal } \\
\text { (ay) }\end{array}$ & $\begin{array}{c}\text { Assento } \\
\text { Vertical } \\
\text { (az) }\end{array}$ & $\begin{array}{l}\text { Assento } \\
\text { Horizontal } \\
\quad(a x)\end{array}$ & $\begin{array}{l}\text { Assento } \\
\text { Horizontal } \\
\text { (ay) }\end{array}$ \\
\hline 1,00 & 0,00158 & 0,00018 & 0,00079 & 0,00187 & 0,00033 & 0,00097 & 0,00147 & 0,00036 & 0,00107 \\
\hline 1,25 & 0,00161 & 0,00020 & 0,00114 & 0,00208 & 0,00038 & 0,00167 & 0,00201 & 0,00054 & 0,00133 \\
\hline 1,60 & 0,00141 & 0,00022 & 0,00148 & 0,00157 & 0,00039 & 0,00183 & 0,00148 & 0,00048 & 0,00166 \\
\hline 2,00 & 0,00095 & 0,00028 & 0,00118 & 0,00103 & 0,00045 & 0,00154 & 0,00114 & 0,00064 & 0,00167 \\
\hline 2,50 & 0,00110 & 0,00035 & 0,00086 & 0,00097 & 0,00056 & 0,00097 & 0,00119 & 0,00084 & 0,00119 \\
\hline 3,16 & 0,00156 & 0,00033 & 0,00077 & 0,00129 & 0,00053 & 0,00073 & 0,00143 & 0,00063 & 0,00082 \\
\hline 4,00 & 0,00138 & 0,00024 & 0,00058 & 0,00139 & 0,00041 & 0,00059 & 0,00171 & 0,00060 & 0,00072 \\
\hline 5,00 & 0,00100 & 0,00015 & 0,00046 & 0,00092 & 0,00021 & 0,00045 & 0,00096 & 0,00025 & 0,00050 \\
\hline 6,00 & 0,00159 & 0,00015 & 0,00074 & 0,00124 & 0,00017 & 0,00061 & 0,00096 & 0,00016 & 0,00052 \\
\hline 8,00 & 0,00109 & 0,00011 & 0,00061 & 0,00163 & 0,00016 & 0,00073 & 0,00234 & 0,00018 & 0,00087 \\
\hline 10,00 & 0,00148 & 0,00010 & 0,00050 & 0,00139 & 0,00010 & 0,00049 & 0,00191 & 0,00011 & 0,00057 \\
\hline 12,50 & 0,00196 & 0,00010 & 0,00055 & 0,00146 & 0,00008 & 0,00052 & 0,00197 & 0,00010 & 0,00072 \\
\hline 16,30 & 0,00099 & 0,00009 & 0,00043 & 0,00107 & 0,00007 & 0,00054 & 0,00126 & 0,00008 & 0,00080 \\
\hline 20,00 & 0,00073 & 0,00008 & 0,00034 & 0,00072 & 0,00006 & 0,00035 & 0,00078 & 0,00007 & 0,00041 \\
\hline 25,00 & 0,00367 & 0,00010 & 0,00067 & 0,00177 & 0,00008 & 0,00041 & 0,00116 & 0,00008 & 0,00043 \\
\hline 31,60 & 0,00259 & 0,00010 & 0,00045 & 0,00450 & 0,00011 & 0,00072 & 0,00404 & 0,00012 & 0,00066 \\
\hline 40,00 & 0,00121 & 0,00009 & 0,00042 & 0,00147 & 0,00009 & 0,00037 & 0,00172 & 0,00009 & 0,00046 \\
\hline 50,00 & 0,00092 & 0,00007 & 0,00071 & 0,00105 & 0,00007 & 0,00064 & 0,00095 & 0,00007 & 0,00071 \\
\hline 63,00 & 0,00088 & 0,00004 & 0,00081 & 0,00106 & 0,00004 & 0,00064 & 0,00101 & 0,00005 & 0,00073 \\
\hline 80,00 & 0,00077 & 0,00003 & 0,00082 & 0,00086 & 0,00003 & 0,00102 & 0,00078 & 0,00003 & 0,00091 \\
\hline 100,00 & 0,00071 & 0,00003 & 0,00155 & 0,00087 & 0,00004 & 0,00234 & 0,00079 & 0,00004 & 0,00228 \\
\hline
\end{tabular}




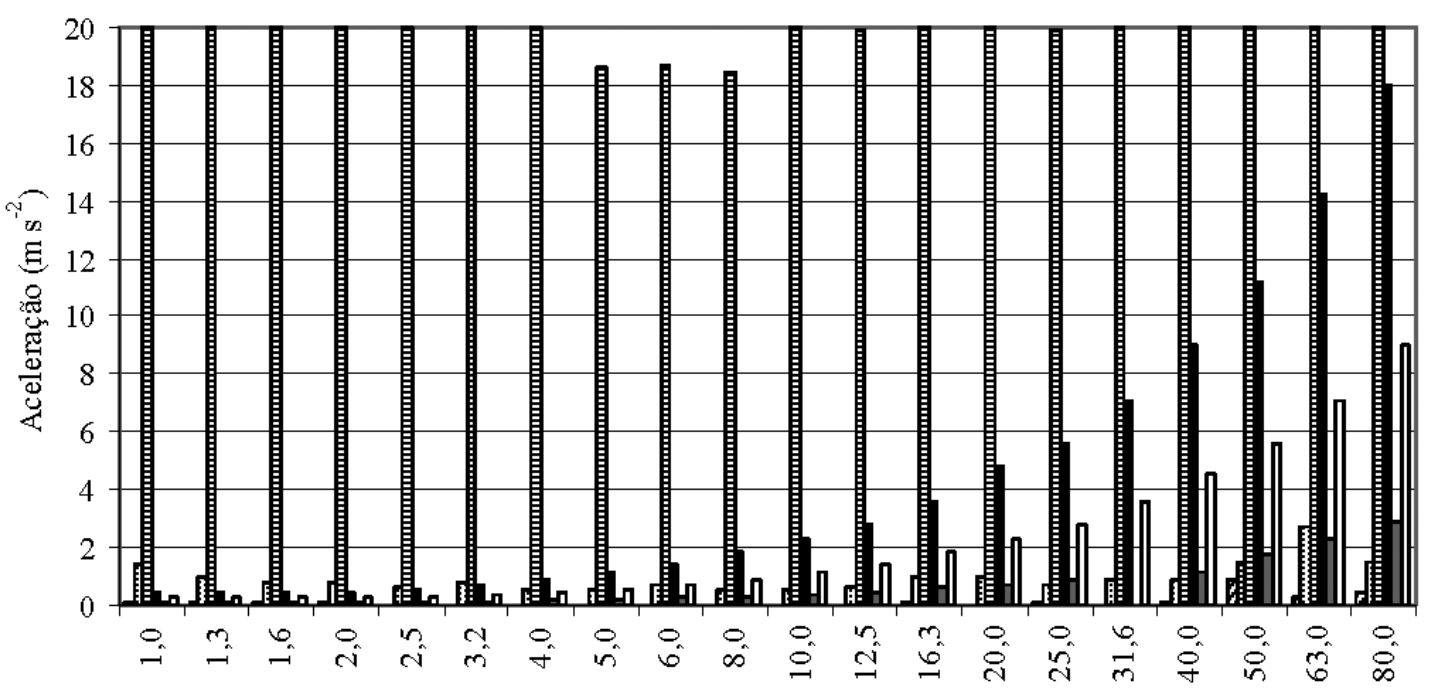

Frequência $(\mathrm{Hz})$

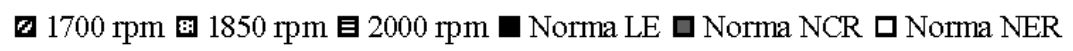

Figura 2. Aceleração RMS (eixo ay) em função da freqüência em bandas de 1/3 de oitava, para as três rotações do trator A (Ano 1988) tracionando o arado, considerando oito horas de trabalho e o permitido pela norma ISO 2631 para limite de exposição (LE), nível de conforto reduzido (NCR) e nível de eficiência reduzida (NER). RMS acceleration (ay-axle) at 1/3rd octave band, during tractor A (1988) operation, under different motor rotations, according to the level permitted by the ISO 2631 Standard (8 hours daily work) for exposure limit (LE), reduced comfort level (NCR) and reduced efficiency level (NER).

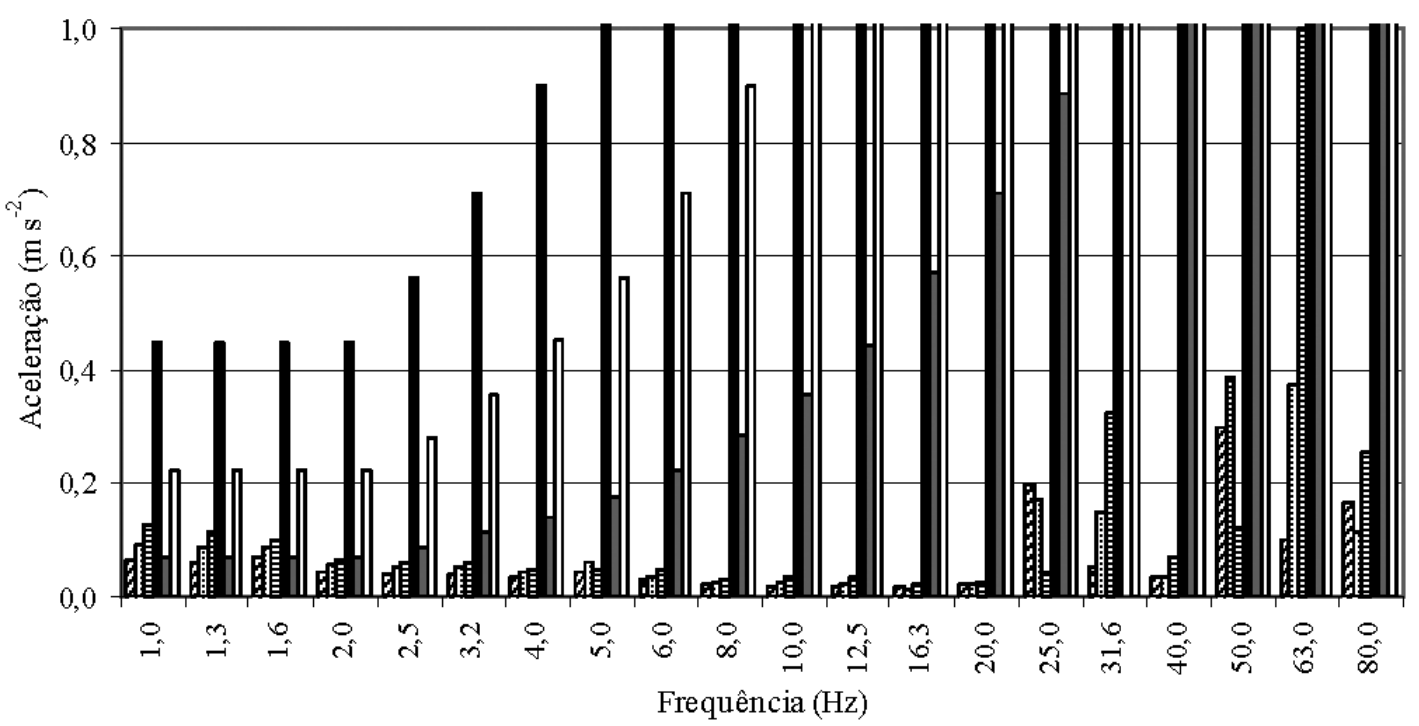

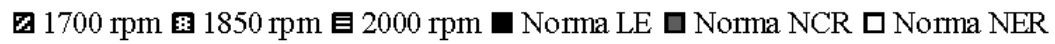

Figura 3. Aceleração RMS (eixo ax) em função da frequiência em bandas de 1/3 de oitava, para as três rotações do trator A (Ano 1988) tracionando o arado, considerando oito horas de trabalho e o permitido pela norma ISO 2631 para limite de exposição (LE), nível de conforto reduzido (NCR) e nível de eficiência reduzida (NER). RMS acceleration (ax-axle) at 1/3rd octave band, during tractor A (1988) operation, under different motor rotations, according to the level permitted by the ISO 2631 Standard (8 hours daily work) for exposure limit (LE), reduced comfort level (NCR) and reduced efficiency level (NER). 


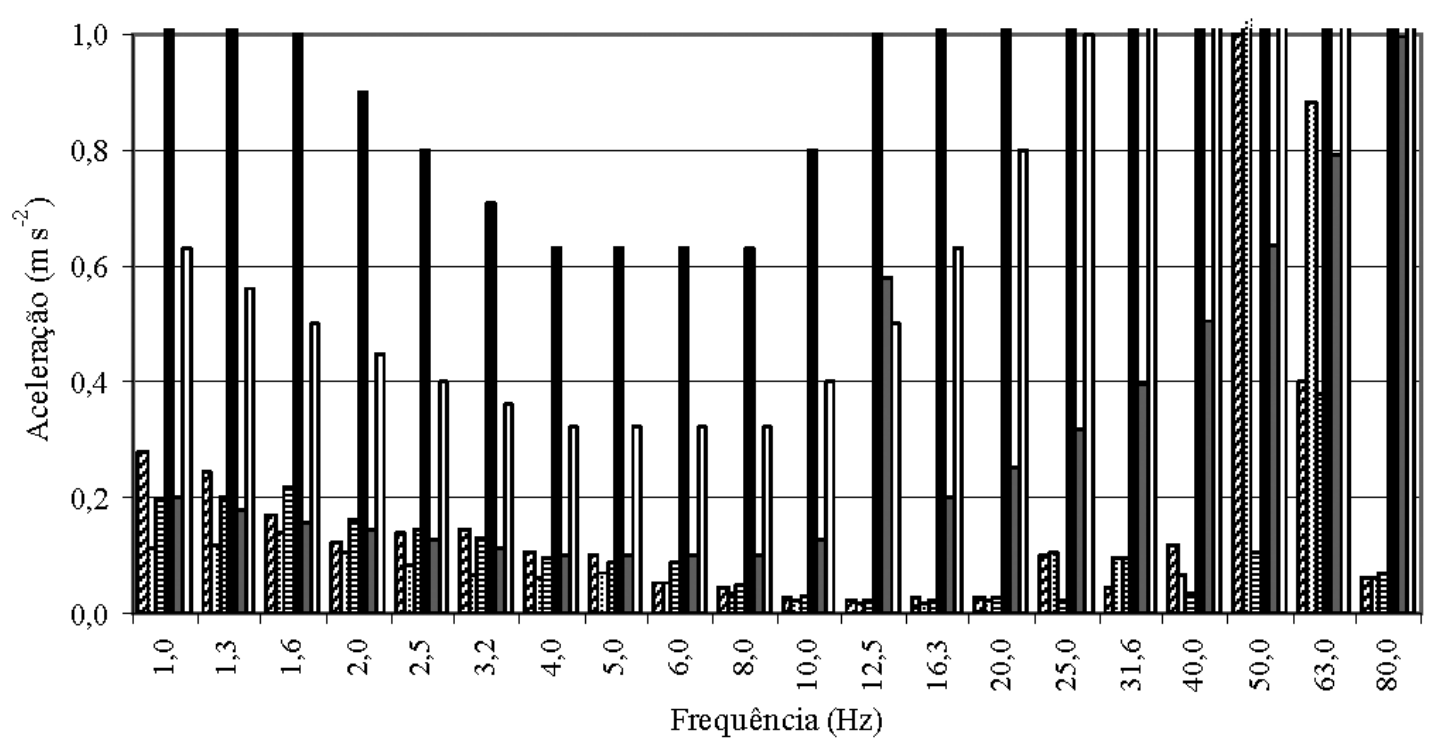

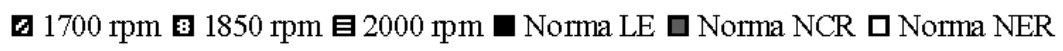

Figura 4. Aceleração RMS (eixo az) em função da frequiência em bandas de 1/3 de oitava, para as três rotações do trator A (Ano 1988) tracionando o arado, considerando oito horas de trabalho e o permitido pela norma ISO 2631 para limite de exposição (LE), nível de conforto reduzido (NCR) e nível de eficiência reduzida (NER). RMS acceleration (az-axle) at 1/3rd octave band, during tractor A (1988) operation, under different motor rotations, according to the level permitted by the ISO 2631 Standard (8 hours daily work) for exposure limit (LE), reduced comfort level (NCR) and reduced efficiency level (NER).

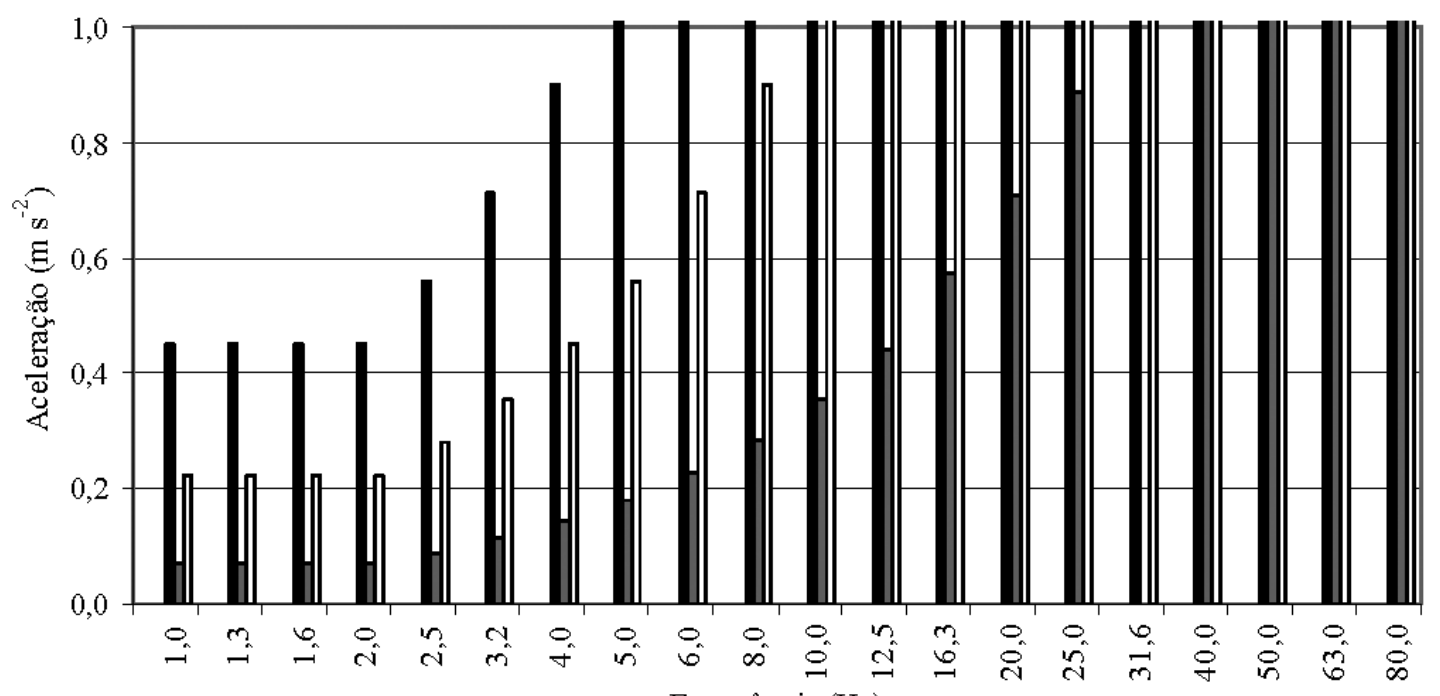

Frequência $(\mathrm{Hz})$

U $1700 \mathrm{rpm}$. $1850 \mathrm{rpm}$ 目 $2000 \mathrm{rpm}$ - Norma LE D Norma NCR Q Norma NER

Figura 5. Aceleração RMS (eixo ay) em função da frequiência em bandas de 1/3 de oitava, para as três rotações do trator B (Ano 2009) tracionando o arado, considerando oito horas de trabalho e o permitido pela norma ISO 2631 para limite de exposição (LE), nível de conforto reduzido (NCR) e nível de eficiência reduzida (NER). RMS acceleration (ay-axle) at 1/3rd octave band, during tractor B (2009) operation, under different motor rotations, according to the level permitted by the ISO 2631 Standard (8 hours daily work) for exposure limit (LE), reduced comfort level (NCR) and reduced efficiency level (NER). 


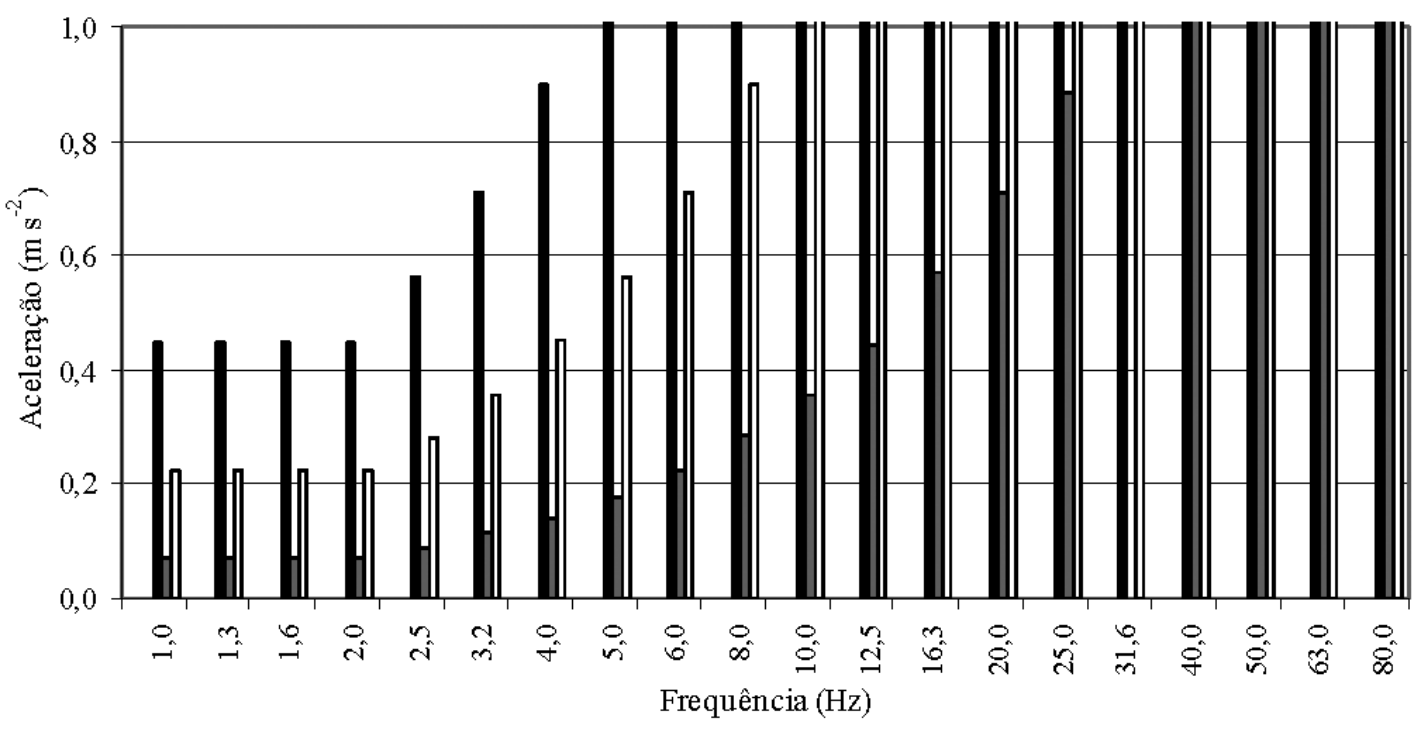

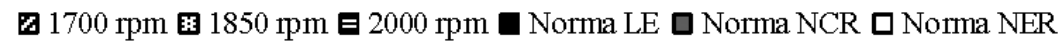

Figura 6. Aceleração RMS (eixo ax) em função da freqüência em bandas de 1/3 de oitava, para as três rotações do trator B (Ano 2009) tracionando o arado, considerando oito horas de trabalho e o permitido pela norma ISO 2631 para limite de exposição (LE), nível de conforto reduzido (NCR) e nível de eficiência reduzida (NER). RMS acceleration (ax-axle) at 1/3rd octave band, during tractor B (2009) operation, under different motor rotations, according to the level permitted by the ISO 2631 Standard ( 8 hours daily work) for exposure limit (LE), reduced comfort level (NCR) and reduced efficiency level (NER).

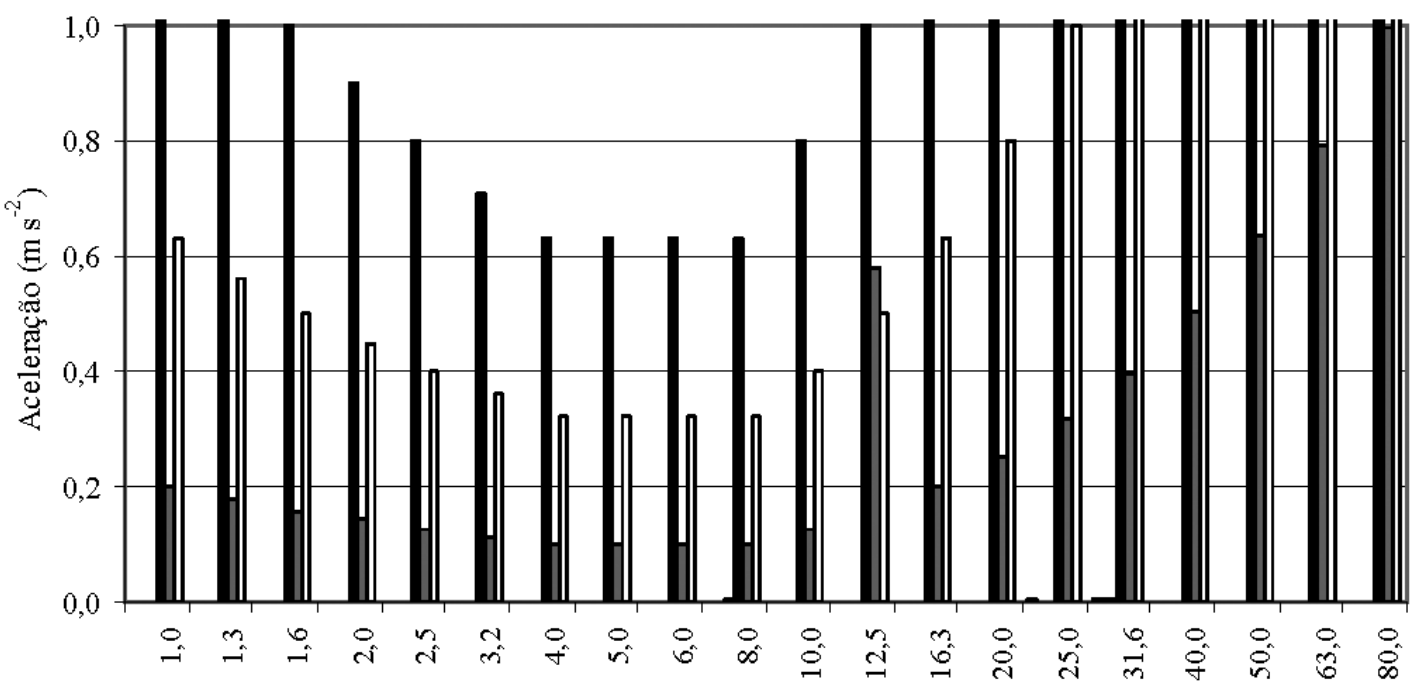

Frequência $(\mathrm{Hz})$

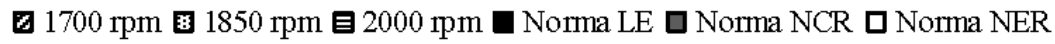

Figura 7. Aceleração RMS (eixo az) em função da freqüência em bandas de 1/3 de oitava, para as três rotações do trator B (Ano 2009) tracionando o arado, considerando oito horas de trabalho e o permitido pela norma ISO 2631 para limite de exposição (LE), nível de conforto reduzido (NCR) e nível de eficiência reduzida (NER). RMS acceleration (az-axle) at 1/3rd octave band, during tractor B (2009) operation, under different motor rotations, according to the level permitted by the ISO 2631 Standard (8 hours daily work) for exposure limit (LE), reduced comfort level (NCR) and reduced efficiency level (NER). 
vibração para o operador, sendo importante conhecer a dinâmica de cada um. A suspensão dos assentos possui mecanismos de molas e amortecedores que produzem uma baixa frequiência de ressonância e isolam vibrações, podendo contribuir com a redução da vibração de corpo inteiro.

Destaca-se que o trator $\mathrm{B}$ possui maior potência que o A, o que também pode ter contribuído, em pequena escala, para atenuar as vibrações, tendo em vista que um motor de maior potência é mais indiferente à variação de carga.

Para o trator A, as piores situações aconteceram no sentido do deslocamento do trator, à medida que se aumentou a rotação do motor. Estes resultados estão de acordo com o trabalho de Scarlett et al. (2007), que encontraram níveis de vibração mais elevados na horizontal do que na vertical ensaiando diversos tratores. Eles atribuem isso a inabilidade da suspensão dos assentos de trator em atenuar a vibração no sentido horizontal. Os autores também citam que a intensidade da vibração está diretamente ligada a operação que está sendo realizada.

\section{Conclusões}

A operação de aração com os tratores avaliados apresentou níveis de ruído, medido junto ao ouvido do operador, acima do limite de $85 \mathrm{~dB}(\mathrm{~A})$ para 8 horas de exposição diária, estabelecido pela NR-15. A grande evolução dos tratores nos últimos anos não foi capaz de reduzir o ruído a níveis passíveis de trabalho sem protetor auricular nos tratores não-cabinados, o que demonstra a necessidade do aprofundamento dos estudos de acústica nesta área e a importância das cabines.

O trator B (ano 2009) apresentou um nível de conforto, em termos de vibração, bem superior ao trator A (ano 1988), demonstrando que o avanço tecnológico, principalmente na qualidade dos sistemas de suspensão dos assentos, foi capaz de reduzir a exposição do operador a níveis de vibração que comprometam sua saúde, segurança, conforto e eficiência. $\mathrm{O}$ trator mais antigo apresentou níveis de vibração acima do permitido pelas normas; o mesmo não ocorreu para o trator mais novo.

\section{Literatura Citada}

Blüthner, R., Hinz, B., Menzel, G., Schust, M., Seidel, H. 2006 On the significance of body mass and vibration magnitude for acceleration transmission of vibration through seats with horizontal suspensions. Journal of Sound and Vibration 298 (4): 627-637.

Bouazara, M., Richard, M. J., Rakheja, S.

2006 Safety and comfort analysis of a 3-D vehicle model with optimal non-linear active seat suspension. Journal of Terramechanics 43: 97-118.

Brasil. Ministério do Trabalho e Emprego

2009 Atividades e operações insalubres. NR-15. Disponível em: < http://www.mte.gov.br/legislacao/normas_regulamentadoras /nr_15.pdf>. Acesso em: 13 jan. 2009.

Debiasi, H., Schlosser, J. F., Pinheiro, E. D.

2004 Desenvolvimento do coeficiente parcial de ergonomia e segurança em tratores agrícolas. Engenharia Agrícola 24 (3): 727-735.

Depczynski, J., Franklin, R. C., Challinor, K., Williams, W., Fragar L. J.

2005 Farm noise emissions during common agricultural activities. Journal of Agricultural Safety and Health 11 (3): 325-334

Dewangan, K. N., Prasanna Kumar, G. V., Tewari, V. K. 2005 Noise characteristics of tractors and health effect on farmers. Applied Acoustics 66 (9): 1049-1062.

Empresa Brasileira De Pesquisa Agropecuária-Embrapa 1999 Sistema brasileiro de classificação de solos. Embrapa Solos. Rio de Janeiro. 306 p.
Franklin, R. C., Depczynski, J., Challinor, K., Williams, W., Fragar, L. J.

2006 Factors affecting farm noise during common agricultural activities. Journal of Agricultural Safety and Health 12 (2): 117-125.

International Organization For Standardization 1978 ISO 2631, Guide for the evaluation of human exposure to whole-body vibration. 2nd. ISO. Geneva. $15 \mathrm{p}$.

Jain, K. K., Shrivastava, A. K., Mehta, C. R. 2008 Analysis of selected tractor seats for seating dimensions in laboratory. Agricultural Engineering International 10 (1): $1-10$.

Marsili, A., Ragni, L., Santoro, G., Servadio, P., Vassalini, G. 2002 Innovative systems to reduce vibrations on agricultural tractors: comparative analysis of acceleration transmitted through the driving seat. Biosystems Engineering 81 (1): 35-47.

Mehta, C. R., Gite, L. P., Pharade, S. C., Majumder, J., Pandey, M. M.

2008 Review of anthropometric considerations for tractor seat design. International Journal of Industrial Ergonomics 38 (5): 546-554.

Paddan, G. S., Griffin, M. J.

2002 Effect of seating on exposures to whole-body vibration in vehicles. Journal of Sound and Vibration 253 (1): 215-241.

Pessina, D.; Guerretti, M.

2000 Effectiveness of hearing protection devices in the hazard reduction of noise from used tractors. Journal of Agricultural Engineering Research 75 (1): 73-80. 
Scarlett, A. J., Price, J. S., Stayner, R. M.

2007 Whole-body vibration: Evaluation of emission and exposure levels arising from agricultural tractors. Journal of Terramechanics 44 (1): 65-73.

Souza, L. H., Vieira, L. B., Fernandes, H. C., Lima, J. S. S. 2004 Níveis de ruído emitidos por uma recolhedora-trilhadora de feijão. Engenharia Agrícola 24 (3): 745-749.
Tewari, V. K., Dewangan, K. N.

2009 Effect of vibration isolators in reduction of work stress during field operation of hand tractor. Biosystems Engineering 103 (2): 146-158

Tiemessen, I. J., Hulsof, C. T. J., Frings-Dresen, M. H. W.

2007 An overview of strategies to reduce whole-body vibration exposure on drivers: A systematic review. International Journal of Industrial Ergonomics 37 (3): 245-25. 